\title{
PHYSIOLOGICAL HYPERBILIRUBINAEMIA IN THE NEWBORN AND THE RESERVOIR FUNCTION OF THE SPLEEN
}

\author{
BY \\ Y. ÅKERRÉN \\ From the Medical Department of the Children's Hospital, Gothenberg, Sweden
}

(Received for Publication June 28, 1950)

In a stimulating article on the genesis of icterus neonatorum Weech (1947) says:

\begin{abstract}
- Physiological hyperbilirubinaemia can be viewed as an explosion which marks the infant's entry in the world ... The sudden change from life in amniotic fluid to life in an atmosphere of air constitutes the trigger which fires the charge in all infants .... An accelerated destruction of haemoglobin begins within a few hours after birth, the destruction apparently being initiated by the onset of pulmonary respiration. The velocity of destruction is more rapid during the first two or three days of life than after the infant is older.'
\end{abstract}

With these words Weech has characterized one of the factors which, according to the hepatohaematogenous theory of the genesis of icterus neonatorum, is a factor in the appearance of physiological hyperbilirubinaemia in the newborn. The other is the hepatic one. The degree of development and the functional capacity of the liver at birth affect the degree and duration of the hyperbilirubinaemia. Many authors, first among them Ylppö (1913), have held that there was insufficient reason to assume increased destruction of erythrocytes and haemoglobin during the neonatal period. However, the majority seem to be agreed that such destruction really occurs. The difficulty of proving quantitatively a parallelism between the degree of hyperbilirubinaemia and the amount of haemoglobin destroyed is largely due, according to Weech, to the functional state of the liver. The degree and duration of the hyperbilirubinaemia depend on the maturity of the liver function at birth. Further, as Weech points out, individual factors also play a part. The supporters of the haemolysis theory appear to be unanimous that the transition from intrauterine hypoxaemia (Barcroft, 1946) to an optimal oxygen supply by the initiation of pulmonary respiration makes the high haemoglobin value per unit of blood functionally superfluous, and thus causes haemolysis. The explanation is teleological.
It affords no answer to the question of the mechanism of the increased destruction of haemoglobin.

Physiological Enlargement of the Spleen during the Neonatal Period

It appeared to me that the investigations concerning physiological enlargement of the spleen in the newborn which I published earlier (Akerrén, 1939, 1942, 1947), might form a starting-point for a theoretical explanation of the assumed increased haemolysis.

In one series daily examinations of the palpability of the spleen were carried out during the whole of the first week of life. In 111 infants $(77 \%)$ the spleen was palpable at one examination at least. In a second series only one examination of the palpability of the spleen was carried out on each baby. In both series all the infants were clinically healthy, born at full-term, and their birth weights were not less than $2,900 \mathrm{~g}$.

The findings in the first series suggested that the spleen was most often palpable on the third day of life, and statistical examination showed that this was not due to chance. In addition the figures showed a trend, first rising to a maximum and then falling. In the second series also the same trend, with a maximum on the third day, was evident.

After the neonatal period the spleen was palpable in $17 \cdot 7 \pm 3 \cdot 6 \%$ of healthy infants less than 3 months old and in $14 \cdot 9-4 \cdot 4 \%$ of children between 3 and 6 months old (Akerrén, 1947). There is a statistical difference between the average frequency with which the spleen is palpable in healthy newborn infants, on the one hand, and in infants at ages between the neonatal period and 6 months, on the other.

The physiological enlargement of the spleen in the newborn is a dynamic, not a static, phenomenon. In the same child the spleen is often palpable one day and not on the following day. Furthermore one can convince oneself in another way that the 
enlargement of the spleen is dynamic, for after an injection of adrenalin it is invariably found that a previously palpable spleen ' disappears'.

\section{The Nature and Causes of the Physiological Enlarge- ment of the Spleen}

The changes in the palpability of the spleen which are met with in the newborn are manifestly due to changes in its volume. In view of their rapid appearance, it must be a mobile substance which causes the swelling of the spleen. Barcroft's classic investigations (1926) have proved that the spleen is a blood reservoir. The blood reservoir is emptied when increased calls are made on the transport of oxygen in the organism, e.g., in physical exertion or hypoxaemia. The reservoir function is reversible. The reservoir fills when functional calls are reduced. It empties under the influence of adrenalin.

During the later part of foetal life there is a marked hypoxaemia (Barcroft, 1946). Probably the high haemoglobin values characteristic of the blood of the newborn are connected with this. With the onset of pulmonary respiration, the hypoxaemia rapidly disappears. It therefore appears extremely probable that blood accumulates in the spleen in the newborn, so that it often becomes palpable. The steady fall in the frequency with which it is palpable after the third day of life, and the lower frequency which characterizes healthy babies after the newborn period, shows that this transitory phenomenon is characteristic.

\section{The Connexion between the Reservoir and Haemolytic Functions of the Spleen}

It has long been thought that the spleen is an organ where erythrocytes are disintegrated and destroyed. In more recent times concrete ideas on the point have emerged from observations or experiments, especially by Fahraeus and his pupils. The point of departure was Fahraeus' (1921) observation that blood with a high sedimentation rate becomes changed when stored at or near body temperature. The sedimentation rate decreases and rouleaux formation is reduced, while the erythrocytes become more spherical. Bergenhem and Fahraeus (1936) showed that this stabilization process is caused by changes both in the plasma (or serum) and in the erythrocytes. The change in the plasma is due to the formation of lysolecithin, which is produced by the action of a lecithinase on the plasma or serum lecithin. The stabilization process is particularly evident when plasma or serum and erythrocytes are again mixed, after having been separated at body temperature. Owing to the change of shape in the direction of spherocytosis undergone by the erythrocytes and the formation of lysolecithin which take place simultaneously, the stabilization process can be regarded as a prelude to haemolysis. (Lysolecithin is the substance to which the strong haemolytic action of cobra venom is due.)

Barcroft's theory of the reservoir function of the spleen drew Fahraeus' attention to the spleen as an organ where an analogous process might take place. He calls the accumulation of blood in the spleen reservoir the 'endopaus' of the blood. Fahraeus (1939) and Bergenhem (1939) pointed out that it was the reduction of the boundary surface between erythrocytes and plasma, appearing during the 'endopaus' which brought about the stabilization process and the accompanying haemolysis or prehaemolysis.

Knisely's investigations (1936 $a$ and b) into the circulation of the blood in the living spleen suggested that when blood entered the sinuses of the spleen from the afferent capillaries, the erythrocytes were continuously stored in them for varying periods. At the same time plasma filtered through the sinus walls into the pulp cords. According to Knisely, the walls of the sinuses acted as a filter, separating red corpuscles from plasma. The conditions for intravital blood separation ('deplasmatization' of the erythrocytes) thus seemed to be present in the spleen.

That changes of a haemolytic or pre-haemolytic nature really occur in the spleen under physiological conditions have been shown in several ways. The venous blood of the spleen, compared with the arterial blood, exhibits both spherocytosis and reduced rouleaux formation (Bergenhem and Fahraeus, 1936; Mellgren, 1939). Mellgren was able to demonstrate haemoglobinaemia in venous blood from the spleen-secured during the emptying of reserve blood from the spleens of catswhich further increased if the blood was stored at body temperature.

Although Fahraeus and his colleagues have given so much experimental support for the assumption that there is a close connexion between the reservoir function of the spleen and its haemolytic function, the theory presupposes that Knisely's conception of the blood circulation in the spleen is correct. Later investigations by Knisely's method have not been confirmed, but at Fahraeus' institute Björkman (1947) has found morphological evidence of a sphincter between sinus and vein. This, Knisely pointed out, is a necessary mechanism for the storage of blood in the sinuses. Björkman has also carried out experiments which appear to prove that separation of erythrocytes and plasma really takes place in the spleen. 
The sinus wall is a rectangular lattice network. In the foetal spleen and in the spleens of newborn infants the mesh is smaller and more circular compared with the rectangular mesh of the adult, a modification which must make the sinus filter finer.

In summary Björkman states:

- The function which is thus maintained is the separation between red cells and plasma. The storiag of erythrocytes occurring during this separation is evidently the same phenomenon as has been interpreted as the splenic reservoir function. The function, however, brings about qualitative changes in the red cells, which, to some extent, reduce their value as an emergency reserve. Because of this fact it seems probable that the reservoir function, as observed by Barcroft and Binet, is only the other side of the shield, and that the essential function is the deplasmatization of the red cells. Its physiological significance has been elucidated by the works of Bergenhem and Fahraeus. It leads to destruction of red corpuscles.

\section{Discassion}

It would seem, therefore. that the reservoir and haemolytic functions of the spleen are identical. The dynamic enlargement of the spleen which makes its appearance during the newborn period is most simply interpreted as a sign of filling of the blood reservoirs. Fahraeus' theory presupposes that there is at the same time increased destruction of haemoglobin and red blood corpuscles respectively. The conception of an increased destruction of blood as one of the main causes of hyperbilirubinaemia in the newborn thus tallies with Fahraeus' theory of physiological haemolysis in the spleen.

According to Weech, destruction of haemoglobin is more rapid during the first two or three days of life than when the infant is older. It is during the same period that I have found the spleen to be palpable with increasing frequency, culminating at about the time when the destruction of haemoglobin is at its height.

\section{Summary}

Weech has compared the onset of pulmonary respiration with the igniting spark which initiates the explosion-like destruction of erythrocytes and haemoglobin in the newborn, which is one of the chief causes, the other being hepatic immaturity, of physiological hyperbilirubinaemia. If an attempt is made to say exactly how the ignition takes place, and where the destruction occurs, the answer appears to be as follows. The onset of pulmonary respiration abolishes intrauterine hypoxaemia. The calls on the means of oxygen transport-the erythrocytes and the haemoglobin - are fewer than before. An unusually large number of red blood corpuscles therefore accumulate in the blood reservoirs of the spleen. This reveals itself in the swelling of the spleen which is characteristic of the newborn period, culminating on about the third day of life. An increased destruction of erythrocytes and haemoglobin begins, owing to the intimate connexion between the reservoir and the haemolytic functions of the spleen, and this in its turn leads to an increase in the bilirubin content of the blood.

\section{REFERENCES}

Ákerrén, Y. (1939). Proc. 7th Northern Pediat. Congr., Oslo, 1938, Acta. Paediatr., Stockh., 24, 23. (1942). Ibid., 29, 395.

(1947). Ibid., 34, 184.

Barcroft, J. (1926). Ergebn. Physiol., 25, 818.

(1946). ' Researches on Pre-Natal Life.' Oxford.

Bergenhem, B. (1939). Acta. Path. Microbiol. Scand., Suppl. 39.

555.

Björkman, S. E. (1947). Acta. med. scand., Suppl. 191. Fahraeus, R. (1921). Ibid., 55, 1. (1939). Lancet, 2, 630.

Knisely, M. H. (1936a). Anat. Rec., 65, 23. (1936b). Ibid.. 65, 131.

Mellgren, J. (1939). J. Physiol., Lond., 94, 483.

Weech, A. A. (1947). Advanc. Pediat., 2, 346.

Ylppö, A. (1913). Z. Kinderheilk., 9, 208. 